\title{
Quantum Critical Behavior of One-Dimensional Soft Bosons in the Continuum
}

\author{
Stefano Rossotti, ${ }^{1}$ Martina Teruzzi, ${ }^{2,1}$ Davide Pini, ${ }^{1}$ Davide Emilio Galli, ${ }^{1}$ and Gianluca Bertaina ${ }^{1, *}$ \\ ${ }^{1}$ Dipartimento di Fisica, Università degli Studi di Milano, Via Celoria 16, I-20133 Milano, Italy \\ ${ }^{2}$ International School for Advanced Studies (SISSA), Via Bonomea 265, I-34136 Trieste, Italy
}

(Received 27 July 2017; published 20 November 2017)

\begin{abstract}
We consider a zero-temperature one-dimensional system of bosons interacting via the soft-shoulder potential in the continuum, typical of dressed Rydberg gases. We employ quantum Monte Carlo simulations, which allow for the exact calculation of imaginary-time correlations, and a stochastic analytic continuation method, to extract the dynamical structure factor. At finite densities, in the weakly interacting homogeneous regime, a rotonic spectrum marks the tendency to clustering. With strong interactions, we indeed observe cluster liquid phases emerging, characterized by the spectrum of a composite harmonic chain. Luttinger theory has to be adapted by changing the reference lattice density field. In both the liquid and cluster liquid phases, we find convincing evidence of a secondary mode, which becomes gapless only at the transition. In that region, we also measure the central charge and observe its increase towards $c=3 / 2$, as recently evaluated in a related extended Bose-Hubbard model, and we note a fast reduction of the Luttinger parameter. For two-particle clusters, we then interpret such observations in terms of the compresence of a Luttinger liquid and a critical transverse Ising model, related to the instability of the reference lattice density field towards coalescence of sites, typical of potentials which are flat at short distances. Even in the absence of a true lattice, we are able to evaluate the spatial correlation function of a suitable pseudospin operator, which manifests ferromagnetic order in the cluster liquid phase, exponential decay in the liquid phase, and algebraic order at criticality.
\end{abstract}

DOI: 10.1103/PhysRevLett.119.215301

Quantum phase transitions (QPTs) [1] play an intriguing role in many-body systems, due to the possibility of unveiling new exotic phases. Progress in the manipulation of ultracold gases allows for the exploration of QPTs, by engineering well-controlled synthetic quantum many-body systems, confined, for example, by optical lattices $[2,3]$ or in quasione-dimensional geometries [4-7]. Recently, Rydberg atoms [8] have emerged as a new route to QPTs $[9,10]$. These are atoms in highly excited electronic states, with a very large electronic cloud. In particular, theoretical [11-14] and experimental [15-17] efforts have focused on ensembles of dressed Rydberg atoms, which are superpositions of the ground state and the above mentioned excited states, coupled via a Rabi process. Their effective interaction can be a softshoulder potential, with a flat repulsion up to a radius $R_{c}$ related to the highly excited state, and a repulsive van der Waals tail at large distances [11,12,18-21]. Quite interestingly, this repulsive interaction belongs to the class that has been recognized to induce cluster formation at high density in classical statistical mechanics [22,23], thanks to the relative freedom of particles at short distances. This has opened a recent flourishing of research on quantum cluster phases: in high dimensions, coexisting cluster crystal and superfluid order have been predicted, yielding supersolid behavior [11-13,24], while in one dimension (1D), cluster Luttinger liquids (CLLs) have been proposed on a lattice [25,26].

In this Letter, we investigate a prototypical system of $N$ bosons in $1 \mathrm{D}$ at linear particle density $n$, governed by the following Hamiltonian in the continuum:

$$
H=-\frac{\hbar^{2}}{2 m} \sum_{i}^{N} \frac{\partial^{2}}{\partial x_{i}^{2}}+\sum_{i<j} \frac{V_{0}}{r_{i j}^{6}+R_{c}^{6}},
$$

where $x_{i}$ are the particle coordinates, $r_{i j}=\left|x_{i}-x_{j}\right|$ the distances, $m$ is the mass, and $V_{0}$ and $R_{c}$ are the strength and the radius of the soft-shoulder potential $V(r)$. If not otherwise specified, in the following we use units of $R_{c}$ for the length, $E_{c}=\hbar^{2} / m R_{c}^{2}$ for the energy, and $\hbar / R_{c}$ for the momenta (see Supplemental Material [27]). The zerotemperature phase diagram (Fig. 1) thus depends on the following two dimensionless quantities: strength, $U=$ $V_{0} /\left(E_{c} R_{c}^{6}\right)$ and density, $\rho=n R_{c}$. By evaluating relevant static and dynamical properties, we show that, while for small $U$ and moderate $\rho$ the system is a Luttinger liquid (LL) [28], although with strong correlation effects, for higher $U$ or $\rho$ a transition occurs towards a CLL. In particular, we focus on the QPT to the dimer cluster liquid, which turns out to be of the 2D Ising universality class. A similar phenomenology has been recently studied in a 1D lattice system governed by the extended Bose-Hubbard Hamiltonian [25,26], while we observe it for the first time in the continuum, where we find that an effective spin Hamiltonian emerges at the transition, even in absence of an underlying lattice.

For generic coupling and density, this system falls into the LL universality class [28], characterized by a gapless bosonic mode at small momenta, with sound velocity $v$. The low-energy and momentum sector of the Hilbert space is governed by the Hamiltonian $H_{\mathrm{LL}}=(v / 2 \pi) \times$ $\int d x\left[K_{L}(\nabla \theta)^{2}+(\nabla \phi)^{2} / K_{L}\right]$, where a large Luttinger 


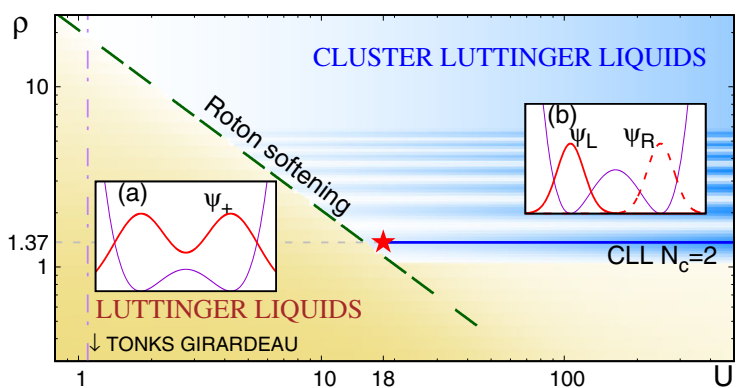

FIG. 1. Phase diagram (log-log scale). A star marks the critical point between the LL and CLL phases for densities commensurate to two-particle clusters. The long-dashed line corresponds to the softening of the Bogoliubov roton. (a) Delocalized orbital in the effective double well. (b) Localized left and right orbitals.

parameter $K_{L}>1$ favors the fluctuations of the particle counting field $\phi(x)$, while small values of $K_{L}$ induce crystal-like behavior, by disordering the phase field $\theta(x)$. The central charge of the associated conformal field theory (CFT) is $c=1$ [29] and for our Galilean invariant system, $v=\rho \pi / K_{L}[28]$.

In the dilute limit, the effects of the interaction are well described by the scattering length $a_{1 \mathrm{D}}$; in particular, for $U \sim 1.09$, we get $a_{1 \mathrm{D}} \sim 0$ [30], corresponding to the TonksGirardeau (TG) model [31]. Conversely, at higher densities, the full shape of $V(r)$ is relevant. Its 1D Fourier transform $\tilde{V}(q)$ [27] features a global minimum at $q_{c} \simeq 4.3$, at which $\tilde{V}\left(q_{c}\right)<0$, providing a typical length $b_{c}=2 \pi / q_{c} \simeq 1.46$. It has been recognized, in the context of classical physics, that such density-independent distance favors clustering, even with a completely repulsive potential [22,23]. Classically, one obtains a $T=0$ cluster crystal, which is destabilized in $1 \mathrm{D}$ by finite temperature, in favor of cluster-dominated liquid phases with different average occupation [32,33]. Quantum mechanics induces coherent delocalization even at $T=0$, rendering the cluster phase a CLL and triggering a QPT towards a LL without cluster order (Fig. 1).

To study the phase diagram in a nonperturbative way, we use the well-established path integral ground state (PIGS) quantum Monte Carlo method [34,35], which represents the ground state as the imaginary-time projection $\exp (-\tau H)\left|\Psi_{T}\right\rangle$ of a trial wave function. We simulate up to $N=200$ particles in a segment of length $L=N / \rho$, using periodic boundary conditions (PBCs). The trial wave function is of the two-body Jastrow form: $\Psi_{T}\left(x_{1}, \ldots, x_{N}\right)=$ $\exp \left\{-\frac{1}{2} \sum_{i<j}\left[u\left(r_{i j}\right)+\chi\left(r_{i j}\right)\right]\right\}$, where $\chi(r)$ accounts for long-wavelength phonons [30,36], while $\exp [-u(r) / 2]$ is the numerical solution of a two-body Schrödinger equation [30], with the effective potential $V_{\text {eff }}(r)=c_{1} V(r)+$ $c_{2} \sum_{l} V(r-l b)$. To reduce projection times, it is crucial to use and optimize this mean-field potential, which accounts for the presence of nearby clusters [27]. We consider excitations associated to density fluctuations, which are commonly investigated via the dynamical structure factor $S(q, \omega)=\int d t\left(e^{i \omega t} / 2 \pi N\right)\left\langle e^{i t H / \hbar} \rho_{q} e^{-i t H / \hbar} \rho_{-q}\right\rangle$. The PIGS algorithm evaluates the numerically exact imaginary-time intermediate scattering function, which yields $S(q, \omega)$ via analytic continuation, through the genetic inversion via falsification of theories algorithm [27,37-40].

We now proceed to discuss our results, first in the LL, then in the CLL regimes. Finally, we discuss the QPT in between the two liquids.

Liquid regime.-Here and in the following, $k_{F}=\pi \rho$ and $E_{F}=k_{F}^{2} / 2$ are the effective Fermi momentum and energy. We concentrate on interaction $U \simeq 1.09$, and increase the density (dot-dashed line in Fig. 1). In the low-density regime $\rho \lesssim 0.1$ [Fig. 2(a)], $S(q, \omega)$ is almost constant, at fixed $q$, in between the particle-hole boundaries $\varepsilon_{\mathrm{IFG}}(q)=\left|k_{F} q \pm q^{2} / 2\right|$, analogously to the $\mathrm{TG}$ gas, which can be mapped to an ideal Fermi gas (IFG). However, within our resolution, the spectral weight has started to gather, especially at the upper boundary, similar to what happens in the Lieb-Liniger model with decreasing coupling parameter [41]. In fact, already at $\rho=0.6$ [panel (b)], the spectrum has evolved into a main mode. As such, it is very well described by the single-peak Feynman approximation $\varepsilon_{\mathrm{FA}}(q)=\varepsilon_{0}(q) / S(q)$, with $\varepsilon_{0}(q)=q^{2} / 2$ the freeparticle energy and $S(q)$ the static structure factor.

By further increasing $\rho$, we simply monitor the evolution of $\varepsilon_{\mathrm{FA}}(q)$ [Fig. 2(c)], and notice that the main excitation becomes more structured, with a roton minimum moving towards $q=q_{c}$. A standard Bogoliubov analysis [11,20]
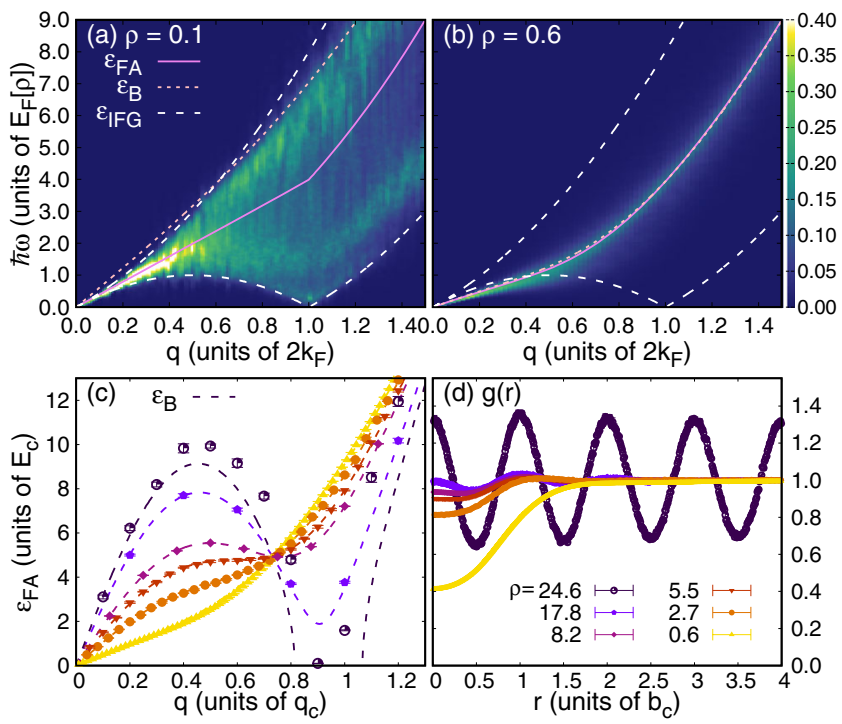

FIG. 2. Dynamical structure factor at $U=1.09$, as a function of momentum $q$ and energy transfer $\varepsilon=\hbar \omega$. Magnitude (color box) is in units of the inverse Fermi energy (values beyond scale are plotted in white). Feynman $\varepsilon_{\mathrm{FA}}$ and Bogoliubov $\varepsilon_{B}$ approximations, and the ideal Fermi gas particle-hole boundaries $\varepsilon_{\mathrm{IFG}}$, are plotted as a reference. Liquid phase at low (a) and intermediate $\rho$ (b). (c) Evolution of $\varepsilon_{\mathrm{FA}}$, compared to $\varepsilon_{B}$. (d) Pair distribution function. 
yields the dispersion $\varepsilon_{B}(q)=\sqrt{\varepsilon_{0}(q)\left[\varepsilon_{0}(q)+2 \rho \tilde{V}(q)\right]}$, which depends only on the combination $\alpha=\rho U$. In this approximation, it is clear that the emergence of the roton minimum is allowed by the momentum dependence of $\tilde{V}(q)$, which has a negative part $[42,43]$. The roton softens at $\alpha=\alpha_{c} \simeq 20.65$. While the agreement between the single-mode $\varepsilon_{\mathrm{FA}}(q)$ and $\varepsilon_{B}(q)$ approximations is very good for $0.6 \lesssim \rho \lesssim 19$, such treatments are in general not valid anymore for $U \gtrsim \alpha_{c} / \rho$ (dashed line in Fig. 1), where indeed our simulations show that clustering occurs.

On increasing $\rho$, the pair distribution function $g(r)$ at first gradually approaches 1 everywhere [Fig. 2(d)], as in classical soft-core fluids in the absence of clustering [44]. However, for very high $\rho$, large-amplitude slowly-decaying oscillations appear, with wavelength $b_{c}$. Again, this behavior is akin to that of classical systems, in the presence of clustering [22]. A Gaussian fit of the peaks indicates, on average, $N_{c} \simeq 36$ particles per cluster at $\rho=24.6$. In the quantum case, the oscillations of $g(r)$ eventually decay as in a cluster liquid, a behavior that we can easily see in the more relevant cluster phases at low $\rho$ and large $U$. In fact, a Hamiltonian description of dressed Rydberg gases is questionable at high $\rho$, due to increased losses to other Rydberg levels in current experiments [27].

Commensurate cluster Luttinger liquid.-We therefore now focus on the density $\rho=2 / b_{c} \simeq 1.37$ (solid line in Fig. 1), commensurate to clusters of $N_{c}=2$ particles [45]. In Fig. 3(a), the PIGS results for $g(r)$ are shown, indicating an evolution to a cluster structure on increasing $U$, with peaks containing two particles. $g(r)$ manifests long-range algebraic decay of the peaks' heights, which demonstrates absence of true crystal order [46]. To interpret these results, we employ CLL theory. In the standard bosonization approach [28], the counting field fluctuates around a lattice with spacing $\rho^{-1}$ : Hamiltonian $H_{\mathrm{LL}}$ is then derived with the assumption that fluctuations are small. However, in a commensurate cluster liquid, clearly fluctuations are small only around a lattice of clusters, with spacing $\rho^{-1} N_{c}=b_{c}$. We follow Refs. [25,26], and obtain the following commensurate CLL form of $g(r)$ :

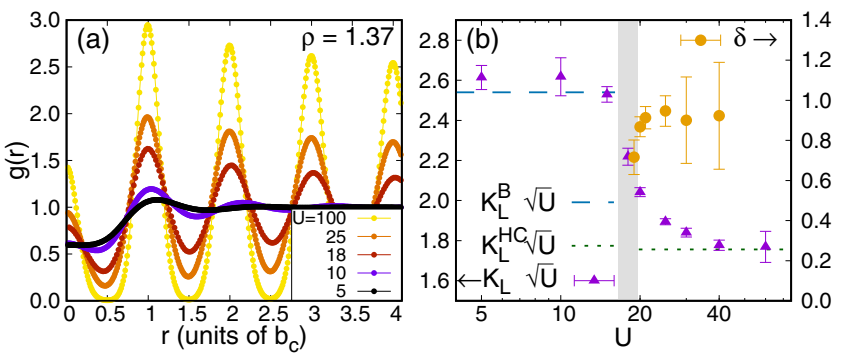

FIG. 3. (a) Pair distribution function at $\rho=1.37$ from the LL to the CLL phases. (b) Left $y$ axis: Luttinger parameter (scaled by $\sqrt{U}$ ) in linear-log scale (triangles), compared to Bogoliubov $K_{L}^{B}=\sqrt{3 \pi \rho / 2 U}$, and harmonic chain $K_{L}^{\mathrm{HC}}=\sqrt{\pi^{2} \rho / b_{c}^{3} \gamma\left(b_{c}\right)}$ predictions. Right $y$ axis: Excess particles per cluster $\delta$ (circles). A band highlights the transition region.

$$
g(r) \underset{r \gg 1 / \rho}{\simeq} 1-\frac{2 K_{L}}{(2 \pi \rho r)^{2}}+\sum_{l=1}^{\infty} A_{l} \frac{\cos \left(2 \pi l \rho r / N_{c}\right)}{r^{2 K_{L}^{\prime} l^{2}}} .
$$

The $r^{-2}$ term is analogue to the standard LL case, while the last term yields dominant density oscillations of wave vector $2 k_{F} / N_{c}=q_{c}$, modulated by an effective Luttinger parameter $K_{L}^{\prime}=K_{L} / N_{c}^{2}$. This implies that, in the CLL phase, the divergence of $S\left(q_{c}\right) \propto N^{1-2 K_{L}^{\prime}}$ is much stronger than what would result from $K_{L}$. We extract $K_{L}$ and $K_{L}^{\prime}$ from the small momentum behavior of $S(q)$ and large distance decay of $g(r)$, respectively [27]. Interestingly, $K_{L}$ scales as $U^{-1 / 2}$ in both the LL and CLL regimes, but with different prefactors. Moreover, we verify that the number of excess particles per cluster $\delta=\sqrt{K_{L} / K_{L}^{\prime}}-1$ quickly goes to 1 for $U>18$ [Fig. 3(b)].

Deep in the cluster phase, a composite harmonic chain (HC) theory can also be envisaged. We write a model Hamiltonian of the type $H_{\mathrm{HC}}=\sum_{i \nu} p_{i, \nu}^{2} / 2+\gamma \sum_{i \nu \mu}\left(x_{i, \nu}-\right.$ $\left.x_{i+1, \mu}\right)^{2} / 2$, where $x_{i, \nu}$ is the displacement of the $\nu$ th particle (with $1 \leq \nu \leq N_{c}$ ) from the average position of cluster $i$ (with $1 \leq i \leq N / N_{c}$ ), and springs of strength $\gamma$ are present only between particles in adjacent clusters, modeling the fact that $V(r)$ is flat at short distances [47]. We obtain centerof-mass modes, of acoustic frequencies $\omega_{\text {acou }}(k)=$ $2 \sqrt{N_{c} \gamma} \sin \left(k b_{c} / 2\right)$, and optical modes, of dispersionless frequency $\omega_{\text {opt }}=\sqrt{2 N_{c} \gamma}$. The latter correspond to relative vibrations of particles in a cluster. We relate $\gamma$ to the meanfield potential felt by a particle if all the others are in a cluster crystal with spacing $b$, and find $\gamma(b)=-\left(4 \pi^{2} / b^{3}\right) \sum_{j=1}^{\infty} \times$ $j^{2} \tilde{V}(2 \pi j / b)$ [48]. It is clear that a stable structure is possible only if $\tilde{V}(2 \pi / b)<0$ for some $b[23,49]$.

In Fig. 4, the spectra at $\rho=1.37$, with decreasing $U$, are shown. Panel (a) $(U=100)$ is deep in the CLL phase: the main peak is in good agreement with the acoustic mode of $\mathrm{HC}$ theory, with $b=b_{c}$. A secondary structure appears at higher frequencies, which we interpret as the optical mode [13]. This is, however, not flat, but strongly modified by anharmonic couplings: these are clearly even more crucial at smaller $U$, where they induce cluster melting.

Ising transition.-The question is now: How are the LL and CLL phases really different? Is the transition simply a crossover? Our data, which show Luttinger liquid behavior on both sides, exclude a Berezinskii-Kosterlitz-Thouless transition to a charge-density-wave, or Peierls transition, even though, at $U=18$, we get $K_{L} \simeq 0.52$ (1) [29]. Moreover, the atomic-pair superfluid transition [50] is also excluded, since, here, formation of larger clusters is allowed and the CLL phase manifests strong quasi-solid order $\left(K_{L}<1 / 2\right)$.

In fact, the physics of this relatively simple system is very rich. The acoustic mode of the CLL phase [Fig. 4, panels (a) and (b)] is gapless at $q=q_{c}$, corresponding to $k_{F}$, at this density. After the transition, to be located at $U=U_{c} \simeq 18$ [panel (c)], this lowest excitation turns into 

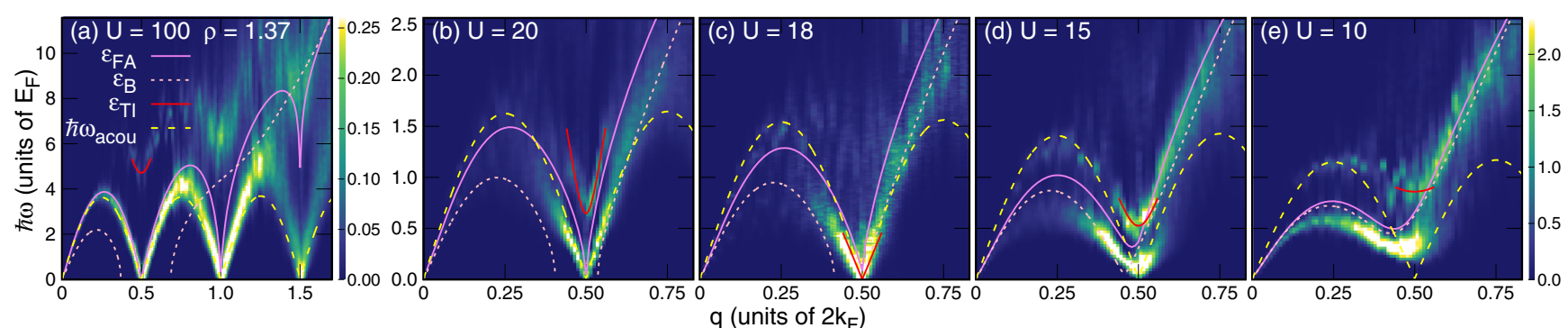

FIG. 4. Spectra at $\rho=1.37$. Panels from (a) to (e) correspond to decreasing $U$, as indicated. Comparison is made to Feynman $\varepsilon_{\mathrm{FA}}$ and Bogoliubov $\varepsilon_{B}$ approximations, and the harmonic chain acoustic mode $\hbar \omega_{\text {acou }}$. At $q \simeq q_{c}$, the secondary mode is fitted by the transverse Ising spectrum $\varepsilon_{\mathrm{TI}}$, Eq. (3). Color scale as in Fig. 2(a).

the rotonic mode [panels (d) and (e)]. Quite interestingly, a weaker secondary mode appears not only in the cluster phase, but also in the strongly correlated liquid phase, in the form of a secondary roton, which connects to the highermomenta main mode. It is reasonable to associate this secondary excitation, in the LL phase, to incipient cluster formation, due to particles being preferentially localized close to either the left or the right neighbor. The crucial observation is that the gap of both such LL excitations [panels (d) and (e)], and the anharmonic optical modes of the CLL phase [(a) and (b)], vanishes at the transition (c), which implies that they proliferate at that point. This behavior is consistent with that of the 1D transverse Ising (TI) model [51-55] of a chain of coupled two-level systems. Its Hamiltonian is $H_{\mathrm{TI}}=-J \sum_{i} \sigma_{i}^{z} \sigma_{i+1}^{z}-h \sum_{i} \sigma_{i}^{x}$, where $\sigma_{i}^{x / z}$ are Pauli matrices at site $i$. It contains both a ferromagnetic coupling $(J>0)$, which forces alignment, and quantum tunneling $(h>0)$ between the eigenstates of $\sigma^{z}$, favoring a paramagnetic state. This model is exactly solvable with a Jordan-Wigner transformation and Bogoliubov diagonalization [56] and yields excitations of energy

$$
\varepsilon_{\mathrm{TI}}(q)=\sqrt{\Delta^{2}+4 J h(\sin q a / 2)^{2}},
$$

where $\Delta=|J-h|$ is the gap and $a$ is lattice spacing, which are gapless only for $h=J$. This signals a QPT from the ferromagnetic to the paramagnetic state, which is dual to the 2D classical thermal Ising transition. In our case, it is natural to associate $\Delta$ to the gap of the secondary mode at $q=q_{c}$, and set $a=b_{c}$, implying that a spin should be identified every two particles. We fit Eq. (3) from our spectra [Fig. 5(a)]: within our accuracy, the behavior of $\Delta$ in $U-U_{c}$ is linear close to the transition, consistent with the dynamical exponent $z=1$ [55]. The point at $U=18$ requires very long projection times: another indication of the presence of a very low-energy mode. Within our resolution, the Luttinger and critical Ising modes have the same velocity at $U=U_{c}$, which would imply low-energy supersymmetry [57].

To corroborate our interpretation, we recall that the central charge $c$ of the critical TI model is $c=1 / 2$, so that, at the transition, the total central charge should be $c=1+1 / 2=3 / 2$, as calculated for the related lattice model [26]. We estimate $c$ from the slope of the energy per particle $\varepsilon(N)=\varepsilon_{\infty}-c E_{F} /\left(6 K_{L} N^{2}\right)$ versus $1 / N^{2}$, employing a standard CFT result for the dominant finite-size effects [58,59]. An increase of $c$ is manifest in Fig. 5(a). It is in fact delicate to extrapolate $c$ close to $U_{c}$ : higher order corrections may become relevant, and field theoretical methods should elucidate the interplay between the Luttinger and Ising fields, as done in Refs. [57,60]. It would be interesting to extract $c$ also from the entanglement entropy, as recently introduced in the PIGS algorithm [61].

It is particularly appealing to investigate the microscopic realization of this effective TI model. The many-body potential surface reduces to double wells as a function of relative distances: two nearby bosons have preferred configurations if they are at $r_{i j}=0$ or $r_{i j} \simeq b_{c}$ [27]. Thus, for each even particle, for example, there are left $\psi_{L}$ and right $\psi_{R}$ preferred cluster configurations, and the potential energy is minimized when subsequent even particles choose the same clustering direction. Anharmonic terms instead give rise to delocalization. The cluster phase is then to be thought of as the ferromagnetic state, where all even particles have chosen either $\psi_{L}$ or $\psi_{R}$ [Fig. 1(b)], while the liquid phase is made of $\psi_{+}=\left(\psi_{L}+\psi_{R}\right) / \sqrt{2}$ states [Fig. 1(a)], where particles continuously hop left and right.

This mapping can be made quantitative, by introducing a simple, but effective string representation of $\sigma^{z}$, inspired by Ref. [54]: first, particles are ordered by their position $k$, and even positions are assigned a lattice index $i=k / 2$; then, a
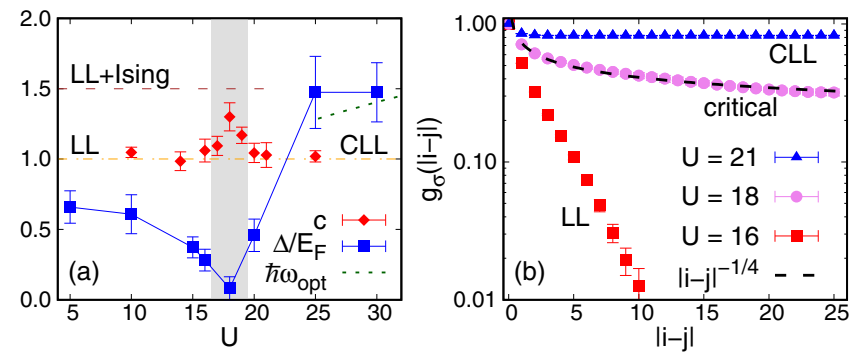

FIG. 5. (a) Second mode gap $\Delta$ (squares), compared to the optical mode energy $\hbar \omega_{\text {opt }}$ (dotted line); central charge $c$ (diamonds). (b) Log-linear scale: pseudospin correlators, compared to the critical behavior (dashed line). 
pseudospin $\sigma_{i}^{z}=1$ is assigned if $\left|x_{k}-x_{k-1}\right|<\left|x_{k}-x_{k+1}\right|$, or $\sigma_{i}^{z}=-1$ in the opposite case. We evaluate the spatial correlator $g_{\sigma}(|i-j|)=\left\langle\sigma_{i}^{z} \sigma_{j}^{z}\right\rangle$ of such a $\mathbb{Z}_{2}$ pseudospin [Fig. 5(b)]. It is very remarkable that $g_{\sigma}$ behaves as expected for the TI model: in the LL (paramagnetic) phase it decays exponentially, while in the CLL (ferromagnetic) phase it manifests true long-range order, which is nonlocal $[54,62,63]$, because of the preliminary ordering of particles. At $U=18$, its behavior is close to an algebraic decay with exponent $\eta \simeq-1 / 4$.

This pseudospin mapping in a continuous system at the LL-CLL transition, as revealed by excitation spectra and a suitable spin correlator, is the key result of this Letter. Such a critical regime could be probed even at finite $T$, given finite experimental sizes. Future work will investigate effects of noncommensurability of $\rho$ with $1 / b_{c}$, which is particularly relevant for trapped gases. Also, an open issue is the presence of quantum Potts transitions at densities commensurate to $N_{C} \geq 3$.

We acknowledge useful discussions with M. Dalmonte, M. Fleischhauer, C. Gross, R. Martinazzo, A. Parola, N. Prokof'ev, and H. Weimer. We acknowledge the CINECA awards IscraC-SOFTDYN-2015 and IscraC-CLUDYN2017 for the availability of high performance computing resources and support. We acknowledge funding from the University of Milan (Grant No. PSR2015-1716LPERI-M).

*gianluca.bertaina@unimi.it

[1] S. Sachdev, Quantum Phase Transitions (Cambridge University Press, Cambridge, England, 2000).

[2] J. Simon, W. S. Bakr, R. Ma, M. E. Tai, P. M. Preiss, and M. Greiner, Nature (London) 472, 307 (2011).

[3] X. Zhang, C.-L. Hung, S.-K. Tung, and C. Chin, Science 335, 1070 (2012).

[4] M. A. Cazalilla, R. Citro, T. Giamarchi, E. Orignac, and M. Rigol, Rev. Mod. Phys. 83, 1405 (2011).

[5] M. Olshanii, Phys. Rev. Lett. 81, 938 (1998).

[6] E. Haller, M. Gustavsson, M. J. Mark, J. G. Danzl, R. Hart, G. Pupillo, and H.-C. Nägerl, Science 325, 1224 (2009).

[7] E. Haller, R. Hart, M. J. Mark, J. G. Danzl, L. Reichsöllner, M. Gustavsson, M. Dalmonte, G. Pupillo, and H.-C. Nägerl, Nature (London) 466, 597 (2010).

[8] R. Löw, H. Weimer, J. Nipper, J. B. Balewski, B. Butscher, H. P. Büchler, and T. Pfau, J. Phys. B 45, 113001 (2012).

[9] H. Weimer, R. Löw, T. Pfau, and H. P. Büchler, Phys. Rev. Lett. 101, 250601 (2008).

[10] P. Schauß, M. Cheneau, M. Endres, T. Fukuhara, S. Hild, A. Omran, T. Pohl, C. Gross, S. Kuhr, and I. Bloch, Nature (London) 491, 87 (2012).

[11] N. Henkel, R. Nath, and T. Pohl, Phys. Rev. Lett. 104, 195302 (2010).

[12] F. Cinti, T. Macrì, W. Lechner, G. Pupillo, and T. Pohl, Nat. Commun. 5, 3235 (2014).

[13] As also suggested in the 2D case in S. Saccani, S. Moroni, and M. Boninsegni, Phys. Rev. Lett. 108, 175301 (2012).
[14] A. Lauer, D. Muth, and M. Fleischhauer, New J. Phys. 14, 095009 (2012).

[15] Y.-Y. Jau, A. M. Hankin, T. Keating, I. H. Deutsch, and G. W. Biedermann, Nat. Phys. 12, 71 (2016).

[16] J. Zeiher, R. van Bijnen, P. Schauß, S. Hild, J.-Y. Choi, T. Pohl, I. Bloch, and C. Gross, Nat. Phys. 12, 1095 (2016).

[17] J. Zeiher, J.-Y. Choi, A. Rubio-Abadal, T. Pohl, R. van Bijnen, I. Bloch, and C. Gross, arXiv:1705.08372.

[18] G. Pupillo, A. Micheli, M. Boninsegni, I. Lesanovsky, and P. Zoller, Phys. Rev. Lett. 104, 223002 (2010).

[19] J. B. Balewski, A. T. Krupp, A. Gaj, S. Hofferberth, R. Löw, and T. Pfau, New J. Phys. 16, 063012 (2014).

[20] T. Macrì, S. Saccani, and F. Cinti, J. Low Temp. Phys. 177, 59 (2014).

[21] M. Płodzień, G. Lochead, J. de Hond, N. J. van Druten, and S. Kokkelmans, Phys. Rev. A 95, 043606 (2017).

[22] C. N. Likos, A. Lang, M. Watzlawek, and H. Löwen, Phys. Rev. E 63, 031206 (2001).

[23] B. M. Mladek, D. Gottwald, G. Kahl, M. Neumann, and C. N. Likos, Phys. Rev. Lett. 96, 045701 (2006).

[24] F. Ancilotto, M. Rossi, and F. Toigo, Phys. Rev. A 88, 033618 (2013).

[25] M. Mattioli, M. Dalmonte, W. Lechner, and G. Pupillo, Phys. Rev. Lett. 111, 165302 (2013).

[26] M. Dalmonte, W. Lechner, Z. Cai, M. Mattioli, A. M. Läuchli, and G. Pupillo, Phys. Rev. B 92, 045106 (2015).

[27] See Supplemental Material at http://link.aps.org/ supplemental/10.1103/PhysRevLett.119.215301 for details on the potential, the experimental realization, the methods, and the extraction of relevant observables.

[28] F. D. M. Haldane, Phys. Rev. Lett. 47, 1840 (1981).

[29] T. Giamarchi, Quantum Physics in One Dimension (Oxford University Press, New York, 2003).

[30] M. Teruzzi, D. E. Galli, and G. Bertaina, J. Low Temp. Phys. 187, 719 (2017). Recall that the definition of the scattering length in 1D implies that it is zero for infinite zero-range repulsion.

[31] M. Girardeau, J. Math. Phys. 1, 516 (1960).

[32] S. Prestipino, Phys. Rev. E 90, 042306 (2014).

[33] S. Prestipino, D. Gazzillo, and N. Tasinato, Phys. Rev. E 92, 022138 (2015).

[34] A. Sarsa, K. E. Schmidt, and W. R. Magro, J. Chem. Phys. 113, 1366 (2000).

[35] M. Rossi, M. Nava, L. Reatto, and D. E. Galli, J. Chem. Phys. 131, 154108 (2009).

[36] L. Reatto and G. Chester, Phys. Rev. 155, 88 (1967).

[37] E. Vitali, M. Rossi, L. Reatto, and D. E. Galli, Phys. Rev. B 82, 174510 (2010).

[38] G. Bertaina, M. Motta, M. Rossi, E. Vitali, and D. E. Galli, Phys. Rev. Lett. 116, 135302 (2016).

[39] M. Motta, E. Vitali, M. Rossi, D. E. Galli, and G. Bertaina, Phys. Rev. A 94, 043627 (2016).

[40] G. Bertaina, D. E. Galli, and E. Vitali, Adv. Phys. X 2, 302 (2017).

[41] J.-S. Caux and P. Calabrese, Phys. Rev. A 74, 031605 (2006).

[42] L. Santos, G. V. Shlyapnikov, and M. Lewenstein, Phys. Rev. Lett. 90, 250403 (2003). 
[43] D. H. J. O’Dell, S. Giovanazzi, and G. Kurizki, Phys. Rev. Lett. 90, 110402 (2003).

[44] A. Lang, C. N. Likos, M. Watzlawek, and H. Löwen, J. Phys. Condens. Matter 12, 5087 (2000).

[45] Conversely, density $\rho=1 / b_{c} \simeq 0.68$ manifests a more standard crossover from $K_{L}>1$ to $K_{L}<1$, not shown here, but see J. Otterbach, M. Moos, D. Muth, and M. Fleischhauer, Phys. Rev. Lett. 111, 113001 (2013).

[46] N. D. Mermin and H. Wagner, Phys. Rev. Lett. 17, 1133 (1966).

[47] PBCs are implied.

[48] Remarkably, discrepancies of this simple expression from the calculation of the normal mode frequencies using the full dynamical matrix, as calculated in T. Neuhaus and C. N. Likos, J. Phys. Condens. Matter 23, 234112 (2011), are very small.

[49] C. N. Likos, B. M. Mladek, D. Gottwald, and G. Kahl, J. Chem. Phys. 126, 224502 (2007).

[50] M. W. J. Romans, R. A. Duine, S. Sachdev, and H. T. C. Stoof, Phys. Rev. Lett. 93, 020405 (2004).

[51] P. Pfeuty, Ann. Phys. (N.Y.) 57, 79 (1970).

[52] R. Coldea, D. A. Tennant, E. M. Wheeler, E. Wawrzynska, D. Prabhakaran, M. Telling, K. Habicht, P. Smeibidl, and K. Kiefer, Science 327, 177 (2010).
[53] E. Shimshoni, G. Morigi, and S. Fishman, Phys. Rev. Lett. 106, 010401 (2011).

[54] J. Ruhman, E. G. Dalla Torre, S. D. Huber, and E. Altman, Phys. Rev. B 85, 125121 (2012).

[55] A. Dutta, G. Aeppli, B. K. Chakrabarti, U. Divakaran, T. F. Rosenbaum, and D. Sen, Quantum Phase Transitions in Transverse Field Spin Models: From Statistical Physics to Quantum Information (Cambridge University Press, Cambridge, England, 2015).

[56] E. Lieb, T. Schultz, and D. Mattis, Ann. Phys. (N.Y.) 16, 407 (1961).

[57] L. Huijse, B. Bauer, and E. Berg, Phys. Rev. Lett. 114, 090404 (2015).

[58] I. Affleck, Phys. Rev. Lett. 56, 746 (1986).

[59] H. W. J. Blöte, J. L. Cardy, and M. P. Nightingale, Phys. Rev. Lett. 56, 742 (1986).

[60] O. Alberton, J. Ruhman, E. Berg, and E. Altman, Phys. Rev. B 95, 075132 (2017).

[61] C. M. Herdman, P.-N. Roy, R. G. Melko, and A. Del Maestro, Phys. Rev. B 94, 064524 (2016).

[62] F. D. M. Haldane, Phys. Rev. Lett. 50, 1153 (1983).

[63] E. G. Dalla Torre, E. Berg, and E. Altman, Phys. Rev. Lett. 97, 260401 (2006). 